\title{
Polarimetry of the Protoplanetary Nebula AFGL 2688
}

\author{
V. G. Klochkova ${ }^{1}$, V. E. Panchuk ${ }^{1}$, M. V. Yushkin ${ }^{1}$, and A. S. Miroshnichenko ${ }^{2,3}$ \\ ${ }^{1}$ Special Astrophysical Observatory, Russian Academy of Sciences, Nizhniü Arkhyz, \\ Karachă̌-Cherkessian Republic, 357147 Russia \\ ${ }^{2}$ Main Astronomical Observatory, Russian Academy of Sciences, Pulkovskoe sh.65, \\ St. Petersburg, 196140 Russia \\ ${ }^{3}$ Ritter Observatory, University of Toledo, Toledo, OH 43606-3390, USA \\ Received September 10, 2003; in final form, November 10, 2003
}

\begin{abstract}
Our spectroscopic (with resolution $R=75000$ ) and spectropolarimetric ( $R=15000$ ) observations with the $6-\mathrm{m}$ telescope of the Special Astrophysical Observatory have enabled us to distinguish photospheric and circumstellar features in the optical spectrum of the bipolar protoplanetary nebula AFGL 2688 for the first time. The linear polarization of the radiation was measured at 5000-6600 $\AA$. The emission in the lines of the sodium doublet and the Swan bands is formed in the envelope, and the mechanism exciting the transitions is resonance fluorescence. We conclude that the circumstellar envelope has a low density. Features of the structure of the nebula are discussed based on published high-angular-resolution photometric and polarimetric (HST NICMOS) data. (c) 2004 MAIK "Nauka/Interperiodica".
\end{abstract}

\section{INTRODUCTION}

Mass loss on and after the asymptotic giant branch (the AGB and post-AGB stages) is accompanied by the formation of a gas and dust envelope. The angular size of this envelope can be below the resolution limit for ground-based telescopes. In this case, asymmetry of the envelope can be detected from the presence of radiation polarized by scattering on the envelope's dust component. The degree of polarization depends on the scattering conditions and the extent to which the star providing the unpolarized light is obscured. The protoplanetary nebula AFGL 2688 is one of three bipolar post-AGB structures resolved by ground-based telescopes [1, $2]$. Thirty years ago, the optical image of the source was thought to represent a pair of compact blue galaxies, IV $\mathrm{Zw} \mathrm{67}$, at a distance of $30 \mathrm{Mpc}$ [3]. During the flights of the AFCRL rockets in 1975, a source that was unusually bright at 10 and $20 \mu \mathrm{m}$ and was not present in the $2.2 \mu \mathrm{m}$ catalog of Neugebauer and Leighton [4] was detected. The nebula's optical image consists of two elliptical lobes with different brightnesses elongated approximately north-south. The central object illuminating the lobes is hidden from the observer by a dark bridge, thought to be a dust disk or torus. High-angular-resolution HST observations [5] have increased the number of objects similar to AFGL 2688 to nine. The parameters and atmospheric chemical composition of the central star were determined in [6]. Here, we discuss published polarimetric data and present the results of new highangular-resolution polarimetric observations aimed at distinguishing the radiation of the central star from that of the circumstellar envelope of AFGL 2688. We selected this object, known (from broadband polarimetry) for its high degree of polarization, for the development of our method.

\section{OBSERVATIONS AND REDUCTION}

Since these are the first high-spectral-resolution spectropolarimetric observations of a post-AGB object, the observational method requires some discussion. Medium resolution is preferable for most spectropolarimetric observations, with the exception of studies of magnetic main-sequence stars, when measurement of the four Stokes parameters within the profiles of magnetically sensitive lines makes it possible to map the stellar magnetic fields $[7,8]$. In other cases, the spectral resolution is chosen based on the fact that, since the polarization of spectral features is low, it is necessary to obtain signal-to-noise ratios for such features appreciably higher than the values characteristic of spectrophotometric measurements of unpolarized light. Within the possibilities provided by a single telescope, this can usually be achieved by reducing the spectral resolution, which is often done without taking into consideration the ratio of the line's width to the detector's resolution. A more detailed consideration shows that the problem cannot be solved so trivially; as an example, we consider the estimation of the uncertainties of equivalent widths, $W$. 


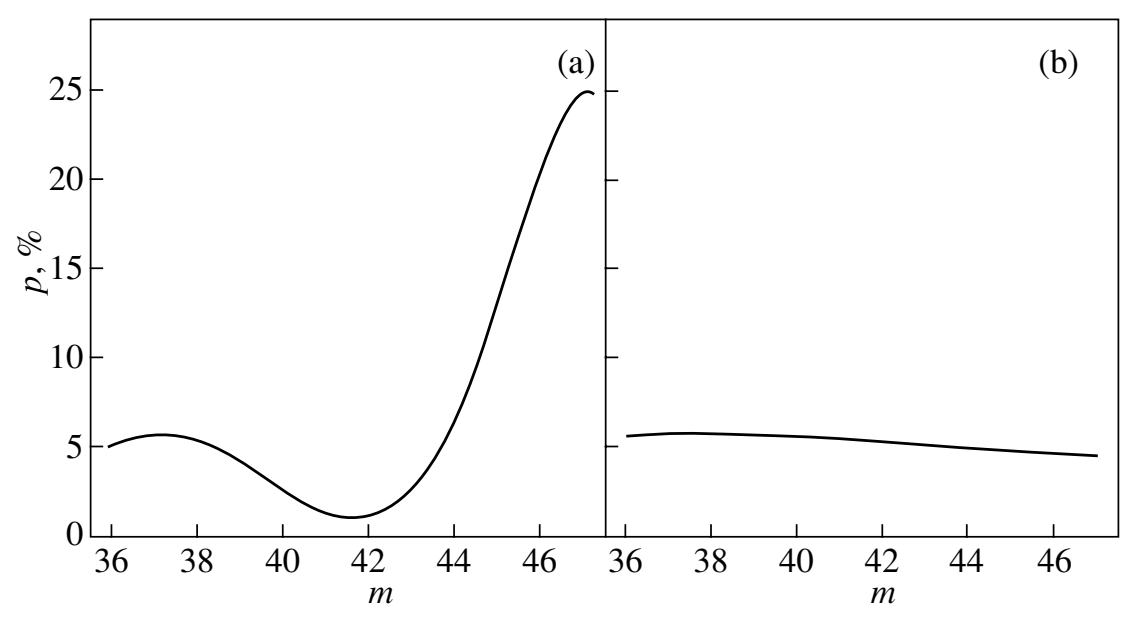

Fig. 1. (a) Instrumental polarization, $p$, measured from spectra of the zero-polarization standard $\zeta$ Peg in the center of each order with number $m$ [11]. (b) Polarization as a function of $m$ from spectral measurements of the polarization standard HD 204827. The instrumental polarization has been subtracted.

For equal numbers of counts, $N$, detected during an exposure per unit wavelength (Ångstrom), the uncertainty in the equivalent width is inversely proportional to the square root of the spectral resolution, $R$, when the light detector's pixel width, $s$, is larger than the line's width, $l$ [9]. Thus, if $s>l$, it is advantageous to increase the spectral resolution, $R$, and not $N$, if the latter must be achieved by increasing the total exposure time. This statement is correct if the readout noise is negligible (as is true for spectrophotometric observations). Thus, the most "economical" spectral resolution (for measurements of $W$ ) is the $R$ value providing a linear resolution equal to the spectral line width $(s=l)$.

Post-AGB stars are surrounded by cool envelopes with narrow lines, and the condition $s<l$ is satisfied only if $R>60000$. For this reason, it is desirable to approach such resolutions if one wishes to use spectropolarimetric observations to separate the light emitted by the star and envelope. Below, we show that increasing the spectral resolution by more than an order of magnitude (from $R<1000$ to $R=15000$ ) made it possible to obtain new spectrophotometric information and enabled us to improve the model of AFGL 2688. However, it is more useful to study the wavelength dependence of the polarization detected for a number of post-AGB objects over a wide spectral range [10] using medium-resolution spectra, since such spectra can also be used to detect (but not measure) polarization effects in individual spectral features.

Our high-spectral-resolution $(R=15000)$ linearpolarization measurements were obtained at the prime focus of the 6-m telescope of the Special Astrophysical Observatory (SAO) with the PFES echelle spectrograph [9] converted into a spectropolarimeter [11]. Observing at the prime focus makes it possible to avoid the effects of instrumental polarization produced at the third (flat) mirror of the $6-\mathrm{m}$ telescope. We simultaneously recorded wavelengths of $4950-6630 \AA$. The width of a pixel of the light detector (a CCD chip with $1160 \times 1040$ elements) corresponded to a radial-velocity interval of $7.7 \mathrm{~km} / \mathrm{s}$. A broadband $(600-700 \mathrm{~nm})$ image of the nebula is shown in Fig. 1 of [1]. We observed the nebula's northern lobe, with the slit center placed on the main axis of the nebula (the line connecting the centers of the optical lobes), $5^{\prime \prime}$ from the nebula's center, which is hidden by a dark bridge. The effective magnitude of the part of the image that fit into the slit was $15.5^{\mathrm{m}}$. During each of the exposures, we rotated the spectrograph to compensate for the rotation of the field that is characteristic of telescopes with altitude-azimuth mounts. During the spectropolarimetric observations, we kept the projection of the slit perpendicular to the nebula's main axis during one exposure, and turned it counterclockwise by $45^{\circ}$ relative to the first position during the next exposure. Examples of the resulting spectropolarimetric echelle images are shown in Fig. 7 of [11]. In addition to the target object, we observed standard stars for which photometric data indicated either high polarization (several percent) or no polarization (zero-polarization standards). The position of the crystal optical axes of the analyzer relative to the spectrograph's elements was always the same.

We reduced the echelle spectrophotometric images with the ECHELLE software package of the MIDAS system using original algorithms tested for the reduction of observations of polarization standards $[11,12]$. In particular, these algorithms make 


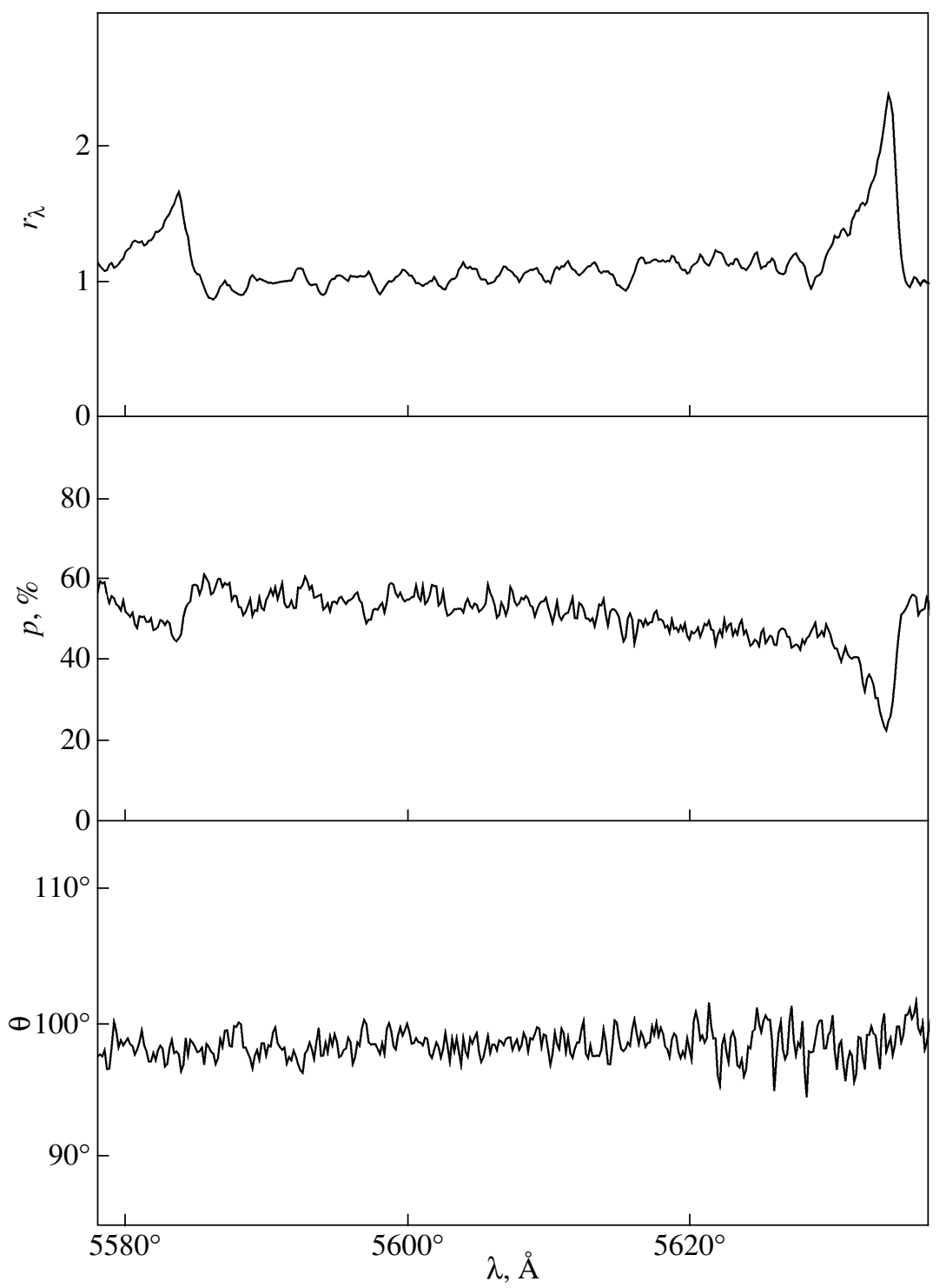

Fig. 2. Fragment of the spectrum $(R=15000)$ of the central part of the northern lobe of the AFGL 2688 nebula near the $\mathrm{C}_{2}$ (1; 2) $5585.2 \AA$ and $(0 ; 1) 5635.5 \AA$ Swan bands and graphs of the linear polarization and polarization position angle.

it possible to remove effects due to the fact that the spectrograph is not rigid, which appear when the projected position angle of the slit is changed by $45^{\circ}$, i.e., during rapid repositioning of the spectrograph. A characteristic feature of spectropolarimetric observations with an echelle spectrograph is the complex relation between the instrumental polarization and the wavelength, which is determined mainly by the mutual position of the diffraction gratings operated in a cross-dispersion mode. For this reason, the planes corresponding to the largest polarization effects from the gratings likewise do not coincide. Together with the wavelength dependence of the polarization, which is different for different gratings, and also because of polarization effects in the antireflection coating of the lens components of the spectrograph optics, this leads to a complex wavelength dependence for the instrumental polarization. Figure la shows the instrumental polarization measured for the centers of the echelle orders during observations of zeropolarization standards. An example of correction for this relation is presented in Fig. 1b, which shows the variation of the polarization with wavelength for the standard HD 204827, whose polarization is known from broadband photometry to be $5.4 \%$ in the $V$ band [13].

Figure 2 shows part of the spectrum of AFGL 2688 near the $(0 ; 1)$ band of the $\mathrm{C}_{2}$ Swan system, along with the calculated parameters of the linear polarization. Note that the scatter in the polarization angle 
is due to the poor statistics of the signal, but the mean angle $\Theta$ is close to the value $99^{\circ} \pm 6^{\circ}$, known from broadband polarimetric observations [1]. When comparing the accuracy of our spectropolarimetric measurements with the broadband polarimetric results, we must keep in mind that the width of each of our spectropolarimetric channels is more than three orders of magnitude smaller than in the case of broadband polarimetric observations. On the other hand, these channels are not sufficiently narrow to enable detailed measurements of the polarization within individual emission features in the spectrum. For example, the residual polarization in the core of the emission components of the sodium doublet (Fig. 3) is partially due to the effects of convolving the object's spectrum with the instrumental function of the PFES spectropolarimeter.

In our analysis, we also use a spectrum of AFGL 2688 taken with the NES quartz echelle spectrograph [14], equipped with an Uppsala CCD chip [15]. The spectrum was acquired with an image slicer [16], providing a resolution of $R=75000$. Fragments of this spectrum were presented in [17], which also gives the systemic radial velocity $(\sim-34 \mathrm{~km} / \mathrm{s})$, the radial velocity measured from photospheric spectral lines $(\sim-15 \mathrm{~km} / \mathrm{s})$, and the radial velocities measured from the envelope's spectrum $(\sim-55 \mathrm{~km} / \mathrm{s})$. The results of our analysis of the star's radial-velocity variations and estimation of the atmospheric line parameters will be presented elsewhere.

\section{DISCUSSION OF THE RESULTS}

We have analyzed the results of our high-spectralresolution spectropolarimetry combined with published photometric, broadband polarimetric, and medium-resolution spectropolarimetric data.

\subsection{Broadband Polarimetry}

Ney et al. [1] present the collected results of the first Johnson $B V R I H K N$ polarimetric measurements. On average, the polarization was about $40 \%$ in the $B V R I$ bands; $31 \%$ in the $H$ band, possibly underestimated due to insufficient sensitivity of the analyzer; $20 \pm 7 \%$ in the $K$ band; and less than $1 \%$ in the $N$ band. The wavelength dependence of the polarization was studied in [18-20]. The linear polarization of the source as a whole at $3700-8800 \AA$ increases linearly with wavelength (from 40 to $50 \%$ ), independent of the size of the entrance diaphragm (in photometric observations of the nebula as a whole, the contribution from the northern lobe dominates). No changes in the polarization were detected in five months of observations.

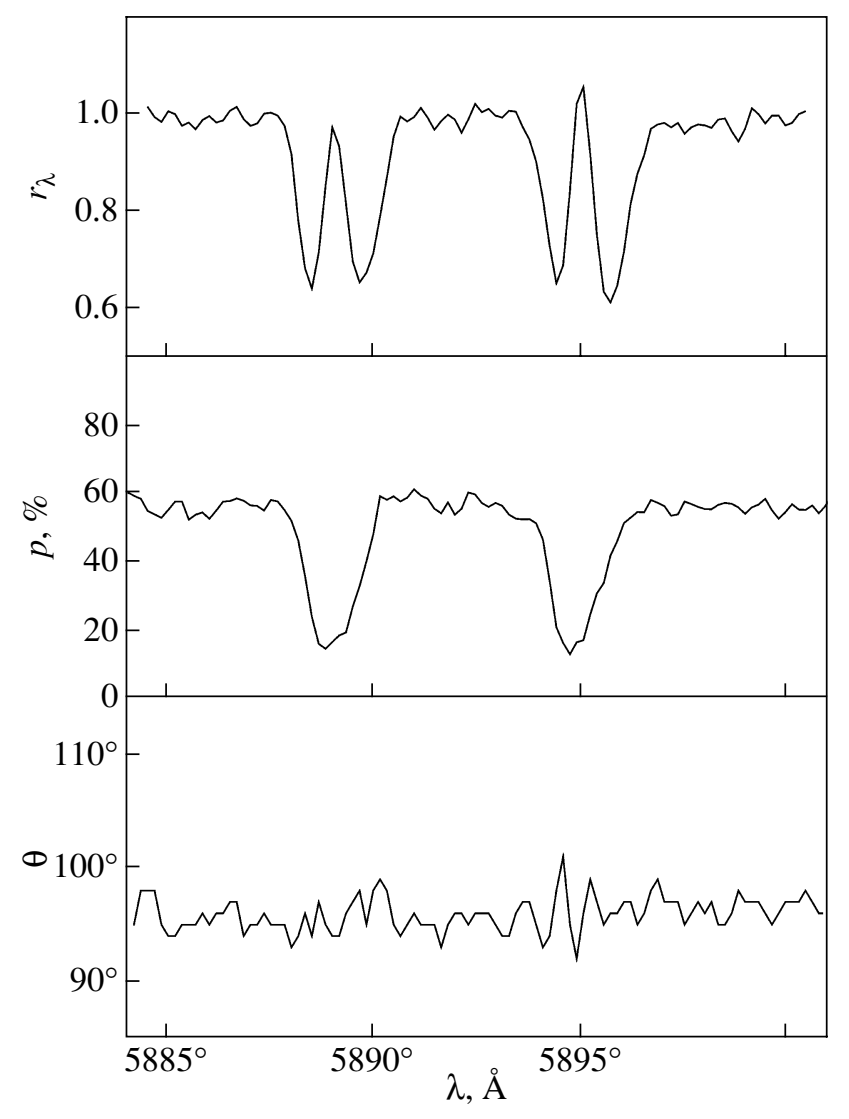

Fig. 3. Same as Fig. 2 for the region of the resonance sodium doublet.

The observations were then compared to a model for polarization induced by interstellar clouds [20] for various ratios of the cloud's depth and length (size in the plane of sky), assuming that the direct light from the star is completely blocked. It was concluded that the observed polarization corresponded to a ratio of the cloud's depth to its length less than $1 / 10$ and that the wavelength dependence of the polarization agreed with the expectations for graphite grains better than those for silicate grains [20]. Measurements at 1.2 and $2.2 \mu \mathrm{m}$ demonstrated that the polarization decreased with wavelength beyond $1 \mu \mathrm{m}$, which can be explained by an increasing contribution from unpolarized thermal emission from the dust envelope [21].

Circular polarization of $-0.67 \%$ that was constant at 3800-7700 $\AA$ was detected in $[18,19]$. This circular polarization could be due to the effects of scattering in the nebula or to the polarizing action of the interstellar medium (ISM). It was demonstrated in a model for scattering by cylinders of infinite length [22] that the wavelength dependences of the circular polarization are very different for different indices of refraction $m$. The modeling demonstrated that, in addition to strong wavelength gradients of the circular 
polarization, a change of the sign of the circular polarization should be observed in the optical. If linearly polarized light passes through an ISM containing aligned anisotropic grains, and if the polarization angle of the light entering the ISM does not coincide with the positions of either of the optical axes of the ISM grains, then a circular-polarization component will be formed. This idea was the basis of the classic study [23], in which the ISM was probed by the passage through it of synchrotron radiation from the Crab nebula [23]. The Crab nebula's intrinsic degree of polarization is the same at different wavelengths, and only the polarization angle is different in different parts of the nebula. The wavelength at which the sign change of the circular polarization in the ISM occurs depends on this angle.

The circular polarization of AFGL 2688 remains the same over a wide wavelength range, so the hypothesis that the circular polarization has an interstellar origin can be rejected. The unusually high linear polarization and the "favorable" orientation of the bipolar structure (the main axis is nearly in the plane of the sky) lead to thoughts of single scattering on nonspherical (elongated) grains that are smaller than the wavelength and are aligned along the nebula's main axis. In this case, the dynamic interaction of the grains with the outflowing gas could provide the alignment mechanism [24]. The predominance of single scattering on small grains contradicts the formation of the circular polarization via repeated scatterings on grains whose size is comparable to the wavelength. Elliptically polarized light can also result from single scattering of unpolarized light on dielectric (with a real index of refraction), nonspherical grains [25]. This problem was considered as a way to explain the elliptic polarization of the zodiacal light (for which the highest degree of circular polarization is $0.86 \%$ [26]), where the multiple-scattering mechanism is also ruled out due to the low concentration of the grains. In this case, the circular polarization will be zero if the symmetry axes of all the grains are perpendicular to the direction of the entering beam and/or are in the scattering plane. If the main axis of the AFGL 2688 nebula is in the plane of the sky, there should be no circular polarization of light scattered on the lobes if the grains are aligned perpendicular to or along the entering beam.

\subsection{Photometry and Polarimetry of Images}

(a) Panoramic optical polarimetry. Broadband polarimetric mapping of the nebula was undertaken at 4500-7000 $\AA$ [27]. The linear polarization of the weak southern lobe $(60 \%)$ was significantly different from that of the bright northern lobe (50\%). For the bipolar nebula $M 1-92$, the polarization of the weak southeastern lobe is $40 \%$, while that of the bright northwestern lobe is $10 \%$ [27], with the relative brightness of the lobes differing more strongly than for AFGL 2688. Comparing the lobe polarizations and surface brightnesses suggests that there is a relation between the lobe brightness and the fraction of light that is singly scattered. The polarization is higher where the fraction of single scatterings (relative to all scatterings) is higher, so that the total number of scatterings, and also the surface brightness, is lower (compared to the opposite lobe with lower polarization, where the contribution from multiple scatterings is higher). An important conclusion follows: the difference in the brightnesses of the lobes of a bipolar nebula demonstrating considerable polarization could be due not to different illumination by the central source but to different numbers of scattering grains, with this effect being more pronounced when the optical depth of the lobes to scattering is low. In this case, the peripheral parts of lobes with lower surface brightness should exhibit higher polarization, as is observed [27]. The lobes' low optical depth to scattering agrees with their low surface brightness: a supergiant with an absolute magnitude of $-6.7^{\mathrm{m}}$ [6] at a distance of $1 \mathrm{kpc}$ has an apparent magnitude of $3^{m}$, whereas the nebula's integrated $V$ magnitude is only $12.2^{m}$ [1]; this drastic difference cannot be explained by interstellar absorption or radiation-dilution effects in the nebula's volume. We can assume that most of the light from the central star passes through the volume of the lobes without hindrance.

(b) Panoramic IR polarimetry. Narrowband, high-angular-resolution HST NICMOS polarimetric measurements were made at $1.99 \mu \mathrm{m}$ to determine the position of the central star illuminating the nebula's lobes [28]. The polarization of the lobes in the near-IR is nearly $10 \%$ higher than in the optical, and the polarization-angle map also demonstrated point symmetry. The source of optical light detected in this way is too far from the compact IR source for them to be considered a close binary that is determining the character of the mass loss of the post-AGB star.

Thus, we can summarize the results of optical and near-IR polarimetry as follows. The grains, thought to be graphite, are small (not larger than $0.1 \mu \mathrm{m}$ ), aspherical, and should be aligned in a direction consistent with the linear- and circular-polarization parameters. The role of interstellar polarization is negligible. Absorption in the lobes can also be neglected. The high polarization at $2 \mu \mathrm{m}$ testifies to an insignificant contribution in the near IR from the dusty component of the lobes themselves.

\subsection{Optical and IR Morphology}

The first models of the nebula explained the brightness difference for the northern and southern lobes of 


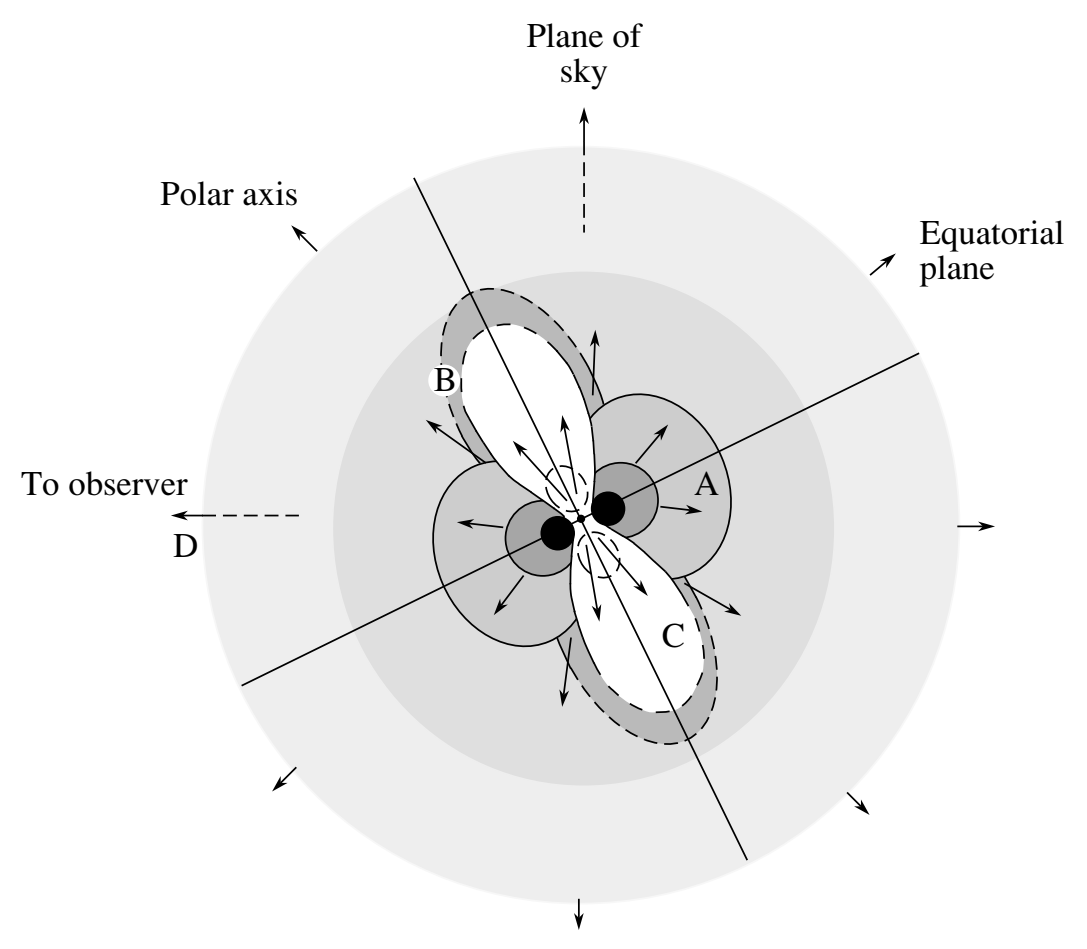

Fig. 4. Classic schematic of the AFGL 2688 [32] nebula. Shown are the disk expanding in the equatorial plane (A), shells expanding in the polar directions (B), wind cavities in these shells (C), and the cold, slowly expanding envelope (D).

AFGL 2688 in the optical as an effect of extinction of the radiation from the southern lobe in the peripheral zone of an extended equatorial dust disk. This interpretation is not consistent with the broadband polarimetric observations (the southern lobe is more strongly polarized); in addition, the small inclination of the disk's axis to the plane of the sky implies a very large size (more than $3000 \mathrm{AU}$ ), and such a disk should have a large mass and angular momentum.

Images of the nebula taken with ground-based instruments show a gradual change in the intensity along the axes of the elliptical lobes, which was interpreted in a model with an axially symmetric, monotonic distribution of the density of the dust component, which is concentrated toward the equator [29]. The slight deviations of the lobes from an elliptical shape-the so-called "horns" (cf., for instance, Fig. 1 in [1])—were modeled by appropriately choosing the gradient for the decrease in the grain density. Such a model, based on multiple scatterings on dust grains, does not explain the high polarization of the lobes of the AFGL 2688 nebula, which was known already after the first broadband photometric observations. A model based on multiple scatterings in the dust envelope assuming a constant scattering optical depth at $0.8-2.2 \mu \mathrm{m}[30]$ remained the main model considered nearly until the HST observations of AFGL 2688 revealed fine structure of the lobes, which were resolved into a complex system of arcs and rays [31].
The horns, for which modeling results were used to identify the inclination of the nebula's axis, are not present in the high-resolution images.

Figure 4 shows a schematic of the nebula taken from [32]. Here, $A$ is the disk expanding in the equatorial plane; $\mathrm{B}$, shells expanding in the polar directions; $\mathrm{C}$, wind cavities in these shells; and D, the cold, slowly expanding envelope. The high polarization of the optical lobes is explained as an effect of reflections from the inner walls of the cavities [32].

The inclination of the nebula's optical axis to the plane of the sky $\left(5^{\circ}-15^{\circ}\right)$, which was used to interpret the spectroscopic measurements in the IR and radio, was obtained in an isotropic scattering model consistent with the flux ratios in the infrared bands and the intensity distribution along the lobes [33]. Additional evidence that this angle is small follows from the "natural" assumption that the nebula's main axis and the plane of the dust disk, which is observed nearly edge-on, are orthogonal.

The high polarization of AFGL 2688 in the optical and IR requires that the dust grains be small, $0.04 \leq$ $a_{0} \leq 0.08 \mu \mathrm{m}$ in size [34]. However, larger grains are needed to explain the centimeter-wavelength radiation from the dust disk detected by the VLA [35]: the spectrum at centimeter and millimeter wavelengths can be modeled by a power-law size distribution for the grains, with an index of 3.5 and the largest diameter of the grains being $a_{C}>0.5 \mathrm{~cm}$. It is thought 
that the small grains are concentrated in the nebula's reflecting lobes, whereas the large grains are concentrated in a dense dust disk lying in position angle $53^{\circ}[35]$.

The dust envelope of AFGL 2688 was modeled in [36] using the refined multiple-scattering mechanism of [30]. It was concluded that the broadband spectrum and the nebula's shape in the optical and IR could not be explained without the presence of larger grains (with radii of at least $5 \mu \mathrm{m}$ ). The spectrum in the optical and up to $3 \mu \mathrm{m}$ is described by "gray" extinction; i.e., this likewise requires that the grains be large. The bipolar structure is clearly visible up to $5 \mu \mathrm{m}$; i.e., the scattering occurs on grains whose size is comparable to the wavelength. The mass of the envelope grains is $4.4 \times 10^{-8} d^{2} M_{\odot}$ or, for a distance of $d=1000 \mathrm{pc}, 4.4 \times 10^{-2} M_{\odot}$. For the gas-todust ratio that is usually adopted for the interstellar medium (158 \pm 13$)$, the envelope's total mass, estimated without taking into account the matter leaving the nebula volume, is $7 M_{\odot}$.

\subsection{Spectropolarimetry}

The first attempt to obtain spectropolarimetric observations with 7 - $\AA$ resolution was undertaken with a 1.8-m telescope [37]. No difference was found in spectra taken at 5400-6800 A for two orthogonal positions of the polaroid. Observations with a 3-m telescope revealed interesting wavelength dependences of the polarization [38]. At 4200-6700 $\AA$ with 7- $\AA$ resolution, high polarization was recorded in the continuum and in strong absorption features (50 and 60\%, respectively, for the nebula's northern and southern lobes), whereas the polarization in the $(0 ; 0),(0 ; 1),(1$; 2) $\mathrm{C}_{2}$ Swan emission bands was lower (by $10-15 \%$ ).

Our observations with the 6-m telescope and spectral resolution more than an order of magnitude higher than in [38] $(R<1000)$ have made it possible to improve the relative contrast of the spectral features, i.e., to reliably separate the spectra formed in the F supergiant's atmosphere and in the extended gas and dust envelope illuminated by the central star.

First, the lobe spectrum demonstrates numerous absorption lines of the supergiant's atmosphere that disappear when the polarization is calculated; i.e., the polarization is the same in the lines and in continuum. This means that the main polarization mechanism acts outside the supergiant's atmosphere. Our observations show that, on average, the polarization in the continuum and photospheric lines of the northern lobe is $52 \%$ in the wavelength range studied.

Second, we find numerous emission bands formed in the circumstellar gas and dust envelope, with polarization minima observed at the positions of these bands (Fig. 2); i.e., the polarization of the emission bands is negligible compared to that of the photospheric light. Thus, the main polarization mechanism acts between the supergiant's photosphere and the formation region of the emission bands (or in that region). Assuming that the emission in the $\mathrm{C}_{2}$ bands is not polarized, we used the energy distributions and polarizations for the most reliably measured bands$(0 ; 0),(1 ; 2)$, and $(0 ; 1)$-to calculate the spectrum of the polarized light. This yielded the continuum level of the photospheric spectrum under the emission bands in the polarized light. Consequently, we can take the characteristics of the photospheric spectrum to be those of the radiation exciting the corresponding molecular transitions when determining whether resonance fluorescence is likely to be acting $[6,39]$.

Third, other details of the wavelength distribution of the polarization were revealed: emission and absorption lines of the sodium resonance doublet, with the polarization near these lines being quite low (Fig. 3). Our analysis taking into account the higherresolution spectrum ( $R=75000)$ demonstrated that these emission lines, which also form in the gas and dust lobe of the nebula, were the doublet's unpolarized component. The continuum polarization is $57 \%$, while the polarization in the doublet line cores is about $13 \%$. Given the observed ratio of the fluxes in the emission and absorption components, these values are consistent with the hypothesis that the emission is unpolarized and that the absorption cores of the doublet's photospheric spectrum has a polarization similar to that of the continuum. We observed sodium line emission at least to distances of $10^{\prime \prime}$ from the center of the northern lobe in the direction of the slit along an east-west line. This means that the lobe width in the sodium emission lines is at least twice the width in the continuum.

Let us discuss the influence of the spectral resolution on the interpretation of the spectrophotometric data, based on a comparison of our data with those of Cohen and Kuhi [38]. Our first comment refers to the $\mathrm{C}_{2}$ bands. Spectrophotometry with medium resolution $(R<1000$ with subsequent smoothing over five channels, yielding an effective $R<300$ [38, Fig. 3]) showed that Swan absorption bands were observed in the polarized radiation. This suggested that these bands were formed before the light was scattered on grains, on the way from the star to the lobe region of the observed nebula. In addition, these absorptions overlap with unpolarized emission formed directly in the lobe. The resulting spectrum consisted of three components: the polarized photospheric spectrum, the envelope's molecular absorption spectrum, and the unpolarized spectrum formed in the lobes. We conclude from our observations $(R=15000)$ that the spectrum in the region of the Swan bands has only 
two components: the F supergiant's photospheric spectrum polarized on the dust component and the unpolarized emission bands. When separating the polarized and unpolarized components, the absorption bands do not stand out in the polarized light, and hence there is no need to place an additional cool molecular envelope between the photosphere and the nebula's lobe. We believe that the appearance of the absorption band in the polarized light is due to the slight (11\%) excess of the emission band's head over the continuum level in [38]. This excess in the high-resolution spectrum is $140 \%(R=15000)$, and our NES observations demonstrate that the excess is preserved when the spectral resolution is further increased (to $R=75000$ ), so that the PFES spectropolarimetry of the Swan bands is already free of the effects of insufficient resolution.

Our second comment deals with the sodium resonance doublet. Strong absorption in the blend of the sodium doublet was revealed in [38] in the spectra of both lobes when observed in polarized light, whereas the spectrum of the northern lobe in unpolarized light shows weak absorption, and the spectrum of the southern lobe, weak emission. Our spectra resolve the doublet, and it is evident that the emission components are at least twice as narrow as the absorption components in the polarized light. The atmospheric absorption lines already demonstrate an asymmetry at $R=15000$. The envelope's emission component provides the unpolarized part of the complex profile. The unpolarized absorption components, which could be formed at the edge of the nebula's lobes as well as in the ISM, equally attenuate the polarized and unpolarized components and, thus, cannot be isolated by means of spectropolarimetry. The contribution from the unpolarized emission component can be identified when the spectrum (Fig. 3a) is compared with the distribution of the polarization (Fig. 3b).

Our third comment concerns the $\mathrm{H} \alpha$ profile. The spectra presented in [38] demonstrate no statistically significant variations of the polarization in the region of $\mathrm{H} \alpha$. Our high-spectral-resolution spectropolarimetry revealed a decrease of the polarization in the absorption core of the $\mathrm{H} \alpha$ line. It is apparent from Fig. 5 that the polarization in the line core is considerably lower, by approximately $15 \%$. The polarization angle does not vary along the $\mathrm{H} \alpha$ profile within the uncertainties.

The profile of the $\mathrm{H} \alpha$ line consists of an absorption component with a narrow core and broad wings and an emission component, which presumably has several parts [6, Fig. 1]. The position of the core corresponds to a radial velocity of $-17 \pm 1 \mathrm{~km} / \mathrm{s}$. The reason for the slight blue shift relative to the velocity system of the photospheric absorption spectrum could be the influence of the $\mathrm{H} \alpha$ line's envelope component, formed on the way from the star's photosphere to the nebula's lobe. Figure 5 shows the profile of the $\mathrm{H} \alpha$ line and the degree and angle of the polarization. We can see that the polarization changes from $50 \%$ in the continuum to $35 \%$ within the line profile; the position of the polarization minimum is nearly coincident with the line's core and is shifted from it only slightly, by $8 \pm 5 \mathrm{~km} / \mathrm{s}$ toward longer wavelengths; and the polarization angle is constant within the errors. The presence of a slight emission component distorting the red wing of the $\mathrm{H} \alpha$ line [6] is not reflected in the polarization variations, providing evidence that the emission has a circumstellar origin; i.e., it originates in the region between the star and the nebula's scattering lobes. The decreased polarization may be due to a higher contribution from unpolarized light, as well as to an increased optical depth that leads to multiple scatterings of the radiation and possible depolarization. There are three possible origins for the polarization minimum in the core of the $\mathrm{H} \alpha$ line: the presence of (i) a narrow unpolarized emission component formed in the core, (ii) an unpolarized emission component whose width exceeds that of the $\mathrm{H} \alpha$ absorption profile, or an unpolarized continuum without lines (for example, due to the envelope's intrinsic thermal emission), or (iii) an absorption component formed after scattering on the grains in the nebula's lobe. Let us consider these three possibilities in more detail.

(1) The narrow unpolarized emission should be formed directly in the nebula's lobe or in outer regions (relative to the lobe), and the matter responsible for the $\mathrm{H} \alpha$ emission should have a line-of-sight projected velocity of $\sim 40 \mathrm{~km} / \mathrm{s}$ away from the observer relative to the system's center of mass. We can estimate the intensity of the hypothetical emission component based on the decrease in the polarization, from $50 \%$ in the continuum to $35 \%$ in the line core, with the residual intensity in the core being 0.45 , resulting in a value of $(10-15) \%$ of the continuum intensity near the $\mathrm{H} \alpha$ line.

(2) In the presence of a broad emission base whose width exceeds that of the $\mathrm{H} \alpha$ absorption wings or in the case of a lineless continuum, the decrease of the polarization follows from the lower contribution of the polarized light in the core of the $\mathrm{H} \alpha$ line compared to the continuum. Consequently, the position of the polarization minimum should coincide with the line core independent of the velocity of the matter forming the unpolarized radiation. We can estimate the intensity of the unpolarized light from the absorption line's depth and the change in the polarization, which is (20-25)\% of the continuum level at the $\mathrm{H} \alpha$ line, while the polarization of the photospheric spectrum should 


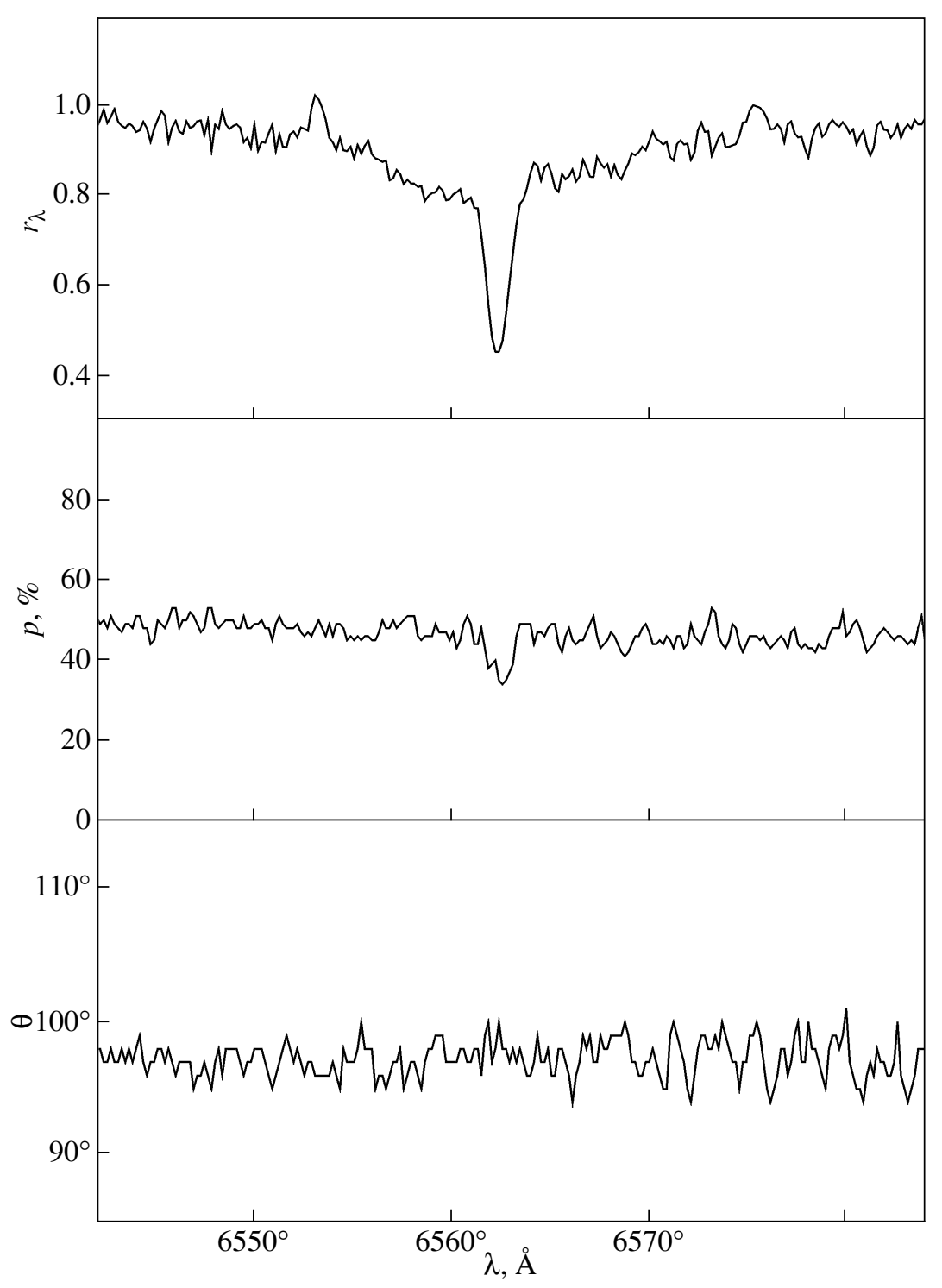

Fig. 5. Same as Fig. 2 for the region of the $\mathrm{H} \alpha$ hydrogen line.

be $\sim 60 \%$. The envelope's intrinsic thermal emission cannot provide this unpolarized radiation, because an even higher continuum polarization is observed in the near-IR. In addition, a broad emission base in the $\mathrm{H} \alpha$ line due to high-velocity motions should have been detected via radio spectroscopic observations. Thus, we reject this interpretation of the polarization minimum in the $\mathrm{H} \alpha$ core.

(3) The polarization in the core of an absorption line formed in the nebula's lobe can also decrease on its way to the observer. The depolarizing factor is resonance scattering of photons on hydrogen atoms. In this case, as in the first possibility, the line-of-sight velocity of the absorbing matter should be $\sim 40 \mathrm{~km} / \mathrm{s}$ away from the observer, relative to the systemic velocity.
Spectropolarimetry of post-AGB stars with spectral resolutions of about $R=1000$ [10] has suggested a division into four types of objects. Based on our observations, AFGL 2688 is a type Ic star: an object whose emission lines and continuum display different polarizations. This effect can arise if the direct light from the star is completely or partially obscured, so that we observe a considerable contribution from the star's scattered polarized light combined with unpolarized radiation from the envelope; alternatively, it is possible that both the star and the emission region are obscured but the polarizations of these components are different due to dilution of the radiation. Our high-resolution spectra, which are the highest quality spectropolarimetric data for a post-AGB star available to date, demonstrate that the emission of AFGL 2688 is essentially unpolarized. 
Thus, our high-spectral-resolution spectrophotometric observations have enabled us to separate the components of the optical radiation formed in the central star's atmosphere and in the bipolar nebula's lobes. Analyzed together with high-angularresolution images acquired with the HST, this suggests a new interpretation for the AFGL 2688 phenomenon.

\subsection{Resonance Fluorescence of the $C_{2}$ Molecules}

(a) The vibrational spectrum of $\mathrm{C}_{2}$. Crampton et al. [37] detected the $\mathrm{C}_{2} 5162 \AA(0 ; 0)$ and $5631 \AA$ $(0 ; 1)$ emission bands but not the $4737 \AA(1 ; 0)$ band, although their blue spectrograms had a resolution of $R=3000$ ( $1.5 \AA)$, a factor of three higher than their red spectrograms $(R=1000,7 \AA)$. Eight emission bands belonging to the $\Delta_{v}=-1$ and $\Delta_{v}=-2$ sequences as well as to the diagonal sequence $\left(\Delta_{v}=\right.$ $0)$, but no bands of the $\Delta_{v}=+1$ sequence, were detected in [6] in a spectrum with $R=15000$. Using our spectrum with $R=75000$ (4653-6080 $\AA$ ), we measured the intensities (in units of the continuum) for the heads of seven emission bands (see the table). We found no trace of the $4737.1 \AA(1 ; 0), 4715.2 \AA(2$; 1), $4697.6 \AA$ ( $3 ; 2), 4684.8 \AA(4 ; 3), 6004.9 \AA(3 ; 5)$, or $6059.7 \AA(2 ; 4)$ bands in the spectrum.

Taking into account the nebula's continuum energy distribution [37], we determined the flux ratios for the heads of the $(1 ; 0),(0 ; 0)$, and $(0 ; 1)$ bands to be $1.2: 3.2: 5.6$ (the continuum fluxes read from Fig. 2 in [37] indicate the flux ratios $1.2: 1.6: 2.5$ ). Calculations of the molecular-band absorption coefficients in a Boltzmann approximation for the level populations $(T=2500 \mathrm{~K})$ using the hypothesis of overlapping lines [40] show that the opacities near the heads of the $(1 ; 0),(0 ; 0)$, and $(0 ; 1)$ bands have the ratios $1.6: 4.0: 0.4$ (the cross sections per grain on a logarithmic scale have the ratios $-16.8:-16.4$ : $-17.4)$. For these bands, the ratios of the FranckCondon factors, $q\left(v^{\prime}, v^{\prime \prime}\right)$, are $0.25: 0.72: 0.22$ [41]. Thus, when the populations of the vibrational levels are in equilibrium, the emission intensity in the (1; 0 ) band should be intermediate between those in the $(0 ; 0)$ and $(0 ; 1)$ bands. We conclude that the vibrational levels of $\mathrm{C}_{2}$ have non-Boltzmann populations and that the intensity behavior of the vibrationalrotational bands repeats the energy distribution in the supergiant's spectrum. This provides evidence for the action of resonance fluorescence.

Note that the resonance-fluorescence hypothesis removes the difficulties encountered by Cohen and Kuhi [42] when explaining the emission-band intensities by invoking the effect of circumstellar reddening.
Relative intensities of the heads of the $\mathrm{C}_{2}$ Swan bands in units of the continuum intensity

\begin{tabular}{c|c|c|c}
\hline$\lambda, \AA$ & $\left(v^{\prime}, v^{\prime \prime}\right)$ & $q$ & $I / I_{c}$ \\
\hline 5097.7 & $(2 ; 2)$ & 0.117 & 1.078 \\
5129.4 & $(1 ; 1)$ & 0.330 & 1.370 \\
5165.2 & $(0 ; 0)$ & 0.724 & 1.988 \\
5501.9 & $(3 ; 4)$ & 0.202 & 1.072 \\
5540.7 & $(2 ; 3)$ & 0.265 & 1.164 \\
5585.2 & $(1 ; 2)$ & 0.286 & 1.723 \\
5635.5 & $(0 ; 1)$ & 0.220 & 2.235 \\
\hline
\end{tabular}

They estimated the extinction in the nebula to be $8.1^{m}$ and $11.5^{m}$ in the directions from the star to the northern and southern lobes, respectively. These estimates do not agree with the extinction derived from the interstellar component of the sodium doublet $\left(4^{m}\right)$ [42], which we believe to be severely overestimated due to an order-of-magnitude error in the use of the equivalent widths of [42] (see [6] for details).

(b) The rotational spectrum of $\mathbf{C}_{2}$. The possible action of resonance fluorescence is also supported by the presence of photospheric absorption lines overlaid by the molecular emission spectrum. For example, the CaI $5581.98 \AA$ line, with a photospheric radial velocity of $-14.8 \mathrm{~km} / \mathrm{s}$, is confidently observed near the head of the $(1 ; 2) 5585.5 \AA$ band, but there is no rotational $\mathrm{C}_{2}$ spectrum near this line. If a different mechanism were exciting the $\mathrm{C}_{2}$ spectrum, this $\mathrm{CaI}$ line should have been overlaid by the $\mathrm{P}_{1}(21)$ rotational emission line. The FeI $5159.06 \AA$ line is observed near the head of the $(0 ; 0) 5165.2 \AA$ band, whereas the blend of the $\mathrm{P}_{1}(27)+\mathrm{P}_{2}(28)$ rotational lines is not seen (although neighboring, weaker lines of the $\mathrm{C}_{2}$ spectrum can be reliably identified).

Our observations with $R=75000$ showed that the mean radial velocity for the heads of four emission bands $(-55 \mathrm{~km} / \mathrm{s})$ is in good agreement with the radial velocity from the emission components of the sodium doublet $(-54 \mathrm{~km} / \mathrm{s})$. This indicates that the kinematic characteristics of the corresponding formation regions are similar; i.e., the regions in which the $\mathrm{C}_{2}$ lines with low rotational quantum numbers, $J$, and the sodium-doublet emission are generated are similar.

The optical spectra of AFGL 2688 can be used to determine if fluorescent equilibrium has been established at the rotational levels of $\mathrm{C}_{2}$. For this purpose, we will make an analogy with the behavior of the $\mathrm{C}_{2}$ rotational lines in the spectra of comets. The $\mathrm{C}_{2}$ molecules in cometary atmospheres are produced by 
the dissociation of $\mathrm{C}_{2} \mathrm{H}_{2}$ molecules, with the formation of $\mathrm{C}_{2} \mathrm{H}$ as an intermediate step; further, the $\mathrm{C}_{2}$ molecules are also photodissociated by the solar radiation. The distance from the region in which the $\mathrm{C}_{2}$ molecules formed to the zone where the statistical equilibrium for $\mathrm{C}_{2}$ is reached is determined by the time scale for achieving equilibrium (10 $\mathrm{min}$ ) and the outflow velocity $(1 \mathrm{~km} / \mathrm{s})$. Adding the distances to the formation zones of the $\mathrm{C}_{2} \mathrm{H}_{2}$ and $\mathrm{C}_{2} \mathrm{H}$ molecules, it is possible to estimate the distance of the maximum $\mathrm{C}_{2}$ concentration from the cometary nucleus. The rotational structure of the $\mathrm{C}_{2}(0 ; 0)$ Swan band in the spectrum of Halley's comet was analyzed in [43]. Two values of the rotational temperature were obtained in a Boltzmann approximation: about $3000 \mathrm{~K}$ for levels with $J>15$ and $190 \mathrm{~K}$ for levels with $J<15$. It was concluded that fluorescence could successfully describe the levels with large $J$ quantum numbers, while the newly formed molecules with low $J$ values had not yet achieved fluorescence equilibrium. If collisional processes are as efficient as fluorescence, the lines have a low rotational temperature, reflecting the kinetic temperature of the gas. It was shown in [44] that a balance between collisional and radiative processes in cometary atmospheres was reached at particle densities of about $10^{10} \mathrm{~cm}^{-3}$; at lower densities, fluorescence equilibrium is achieved. The conditions in the envelopes of protoplanetary nebulas differ from those in cometary heads in the following aspects. First, there are two sources of photodissociation: the central star's light and external UV radiation from the ISM. Second, the outflow velocities are an order of magnitude higher, although the role of this factor is negligible because of the large distance to the object (the distance covered by the newly formed molecules before they reach the zone of statistical equilibrium is within the projected width of the spectrograph's slit). If the fraction of newly formed $\mathrm{C}_{2}$ molecules is high for the observed part of the lobe and collisional processes are significant, the low- $J$ levels should have their Boltzmann populations for a temperature that is close to the gas temperature. The low- $J$ rotational lines whose positions coincide with the photospheric absorptions (with the Doppler shift taken into account) should have Boltzmann intensities, i.e., emission lines that are positionally coincident with photospheric absorption lines should not be weakened, since the fluorescence processes are predominant. Near the heads of the $\mathrm{C}_{2}$ bands in the spectrum of AFGL 2688, we observe deviations of the rotationalline intensities from a Boltzmann distribution due to photospheric absorption, i.e., to a deficiency of illumination. This suggests that fluorescent equilibrium has been achieved and that the gas in the lobe has a low density (below $10^{10} \mathrm{~cm}^{-3}$ ).
Thus, the ratio of the intensities of the vibrational and rotational bands in the $\mathrm{C}_{2}$ spectrum provides further evidence for a low gas density in the lobe. This density can be made considerably lower if we estimate the mass of gas in the nebula's envelope. Let us suppose that only stars with main-sequence masses not exceeding $8 M_{\odot}$ can reach the post-AGB stage. We can calculate the nebula volume from the angular size assuming a distance of $1 \mathrm{kpc}$. If all the "excess" mass was ejected but did not leave the volume, we obtain a maximum density that is at least five orders of magnitude lower than $10^{10} \mathrm{~cm}^{-3}$. This represents additional evidence for the action of resonance fluorescence and for a high transparency of the lobes.

\subsection{Character of the Matter Distribution}

What determines the shape of the optical lobesthe character of the nebula's illumination or the distribution of the scattering material? High-angularresolution images reveal quasi-concentric luminous arches reaching as far as the plane of the dark bridge [31]. This suggests that the solid angle within which the nebula material is illuminated is large $\left(\sim 170^{\circ}\right.$, projected onto the plane of the sky). The arches apparently reflect the nonmonotonic character of the envelope's ejection during the AGB stage, and we can accordingly assume that the light from the arches is due to gas-dynamical effects and not to scattering of the illuminating radiation. However, the quasi-concentric arches do not show the ellipticity (1.6-1.3) that follows from models for the latitude variations of the gas density and of the drift velocity of the dust grains [29,30]. Similar arches have been found for objects with larger inclinations for their main axes [45].

The optical lobes glow predominantly in the continuum (the contribution from $\mathrm{C}_{2}$ and $\mathrm{NaI}$ emission is negligible). Thus, the lobe region contains continuum-scattering material, and since the scatterings are single, it is natural to assume that there is virtually no scattering material outside the lobes. The lobes consist of numerous radial rays that can be traced, like the regions of shadow between them, to distances of tens of arcseconds [31], confirming that the star's light undergoes single scattering on the dust component of the envelope (the contrast of the rays would be lower in the case of multiple scatterings). The polarization plane is perpendicular to the rays $[27,28]$, indicating that the polarizing (scattering) agent is related to the rays. This possibility is also supported by the higher contrast of the rays in the southern lobe [31], where the polarization is higher [27]. The lobes are the brightest in regions with a higher density of rays. The polarization in regions between the rays is lower than in the rays (cf. the 
image of the inner part of the southern lobe, Fig. 1 in [28]).

The presence of long, narrow, high-contrast rays is inconsistent with multiple-scattering models. A fairly exotic "searchlight" scheme was suggested to explain the existence of such narrow rays $\left(\sim 0.1^{\prime \prime}\right)$ : the star is in a cocoon with near-polar windows that form annular holes that let the narrow beams escape [31]. In this picture, the brightnesses of the lobes differ because the cocoon with near-polar windows surrounding the star has a larger optical depth in the direction of the southern lobe than in the direction of the northern lobe. When interpreting the HST images, it was supposed [31] that the existence of the dark inter-ray space did not prove the absence of scattering material there, since the first millimeter observations in CO lines showed a spherically symmetric, uniform distribution of matter in the nebula volume. However, high-angular-resolution interferometric observations have since been obtained and provide evidence that the nebula volume is filled nonuniformly with CO molecules [46]. In our opinion, the weak point in the searchlight scheme is the assumption that the volumes occupied by the luminous rays contain matter identical to that in the dark inter-ray space. The alternative possibility that scattering material is present in the region of the luminous rays and absent from the dark regions is equally plausible. In any case, precisely this picture is observed just beyond the outer boundaries of the lobes (the resonance sodiumdoublet emission is observed in a much larger volume than the scattering of the photospheric spectrum). In a scheme in which the scattering material is restricted to the radial rays and concentric arches, there is no need to look for a mechanism operating near the central star that collimates the beams of light.

Our proposed picture, in which both hemispheres of the nebula are nearly completely illuminated by the central star (with the exception of the plane of the dark bridge), differs from models with wide, partially shaded cones [47] or a certain latitude dust distribution $[29,30,36]$. For this reason, estimates of the envelope mass based on the dust component's mass can be lowered.

\section{CONCLUSIONS}

We have carried out optical spectropolarimetry of AFGL 2688 with a resolution a factor of 50 better than those of previously published data. This has enabled us to distinguish the spectra of the central star and of the circumstellar envelope. The absorption lines in the spectrum of the central star have the same degree polarization as the photospheric continuum. Seven $\mathrm{C}_{2}$ Swan emission bands, emission components of the $\mathrm{NaI}$ resonance doublet, and a narrow
$\mathrm{H} \alpha$ component were identified in the envelope's spectrum. We have demonstrated that resonance fluorescence could excite the $\mathrm{C}_{2}$ spectrum and conclude that the density of the nebula's lobes is low. Based on the results of polarimetric, high-spectralresolution spectropolarimetric, and high-angularresolution photometric and polarimetric observations, we have suggested a new picture for the nebula's structure.

When successfully applied, high-spectral-resolution polarimetric observations can be used to reveal gas and dust envelopes around protoplanetary nebulas observed from the ground with relatively low angular resolution.

\section{ACKNOWLEDGMENTS}

This study was supported by the Russian Foundation for Basic Research (project code 02-02-16085), the Federal Program "Astronomy," and the basic research program of the Physical Sciences Department of the Russian Academy of Sciences "Extended Objects in the Universe" (subproject "Spectroscopy of Extended Envelopes of Evolved Stars"). V.G.K. and M.V.Yu. were also supported by a grant from the President of Russia for young scientists (MK874.2004.2).

\section{REFERENCES}

1. E. P. Ney, K. M. Merrill, E. E. Becklin, et al., Astrophys. J. 198, L129 (1975).

2. S. Kwok, B. J. Hrivnak, C. Y. Zhang, and P. P. Langill, Astrophys. J. 472, 287 (1996).

3. N. Carozzi, P. Chamaraux, and R. Duflot, Astron. Astrophys. 33, 113 (1974).

4. G. Neugebauer and R. B. Leighton, Two-Micron Sky Survey. A Preliminary Catalog (NASA, Washington, 1996), p. 309.

5. K. Y. L. Su, B. J. Hrivnak, and S. Kwok, Astron. J. $122,1525(2001)$.

6. V. G. Klochkova, R. Szczerba, and V. E. Panchuk, Pis'ma Astron. Zh. 26, 510 (2000) [Astron. Lett. 26, $88(2000)]$.

7. N. E. Piskunov, in Magnetic Fields of Chemically Peculiar and Related Stars, Ed. by Yu. V. Glagolevskij and I. I. Romanyuk (Moscow, 2000), p. 96.

8. O. P. Kochukhov, in Magnetic Fields of Chemically Peculiar and Related Stars, Ed. by Yu. V. Glagolevskij and I. I. Romanyuk (Moscow, 2000), p. 106.

9. V. E. Panchuk, I. D. Najdenov, V. G. Klochkova, et al., Bull. Spec. Astrophys. Observ. 44, 127 (1998).

10. S. R. Trammel, H. L. Dinerstein, and R. W. Goodrich, Astron. J. 108, 984 (1994).

11. V. E. Panchuk, V. G. Klochkova, M. V. Yushkin, et al., SAO Preprint No. 159 (Special Astrophysical Observatory, 2001). 
12. M. V. Yushkin, Candidate's Dissertation in Physics and Mathematics (Spets. Astrofiz. Obs. Ross. Akad. Nauk, Nizhniǔ Arkhyz, 2002), p. 182.

13. D. A. Turnshek, R. C. Bohlin, R. L. Williamson II, et al., Astron. J. 99, 1243 (1990).

14. V. E. Panchuk, V. G. Klochkova, and I. D. Naŭdenov, SAO Preprint No. 135 (Special Astrophysical Observatory, 1999).

15. V. E. Panchuk, N. E, Piskunov, V. G. Klochkova, et al., SAO Preprint No. 169 (Special Astrophysical Observatory, 2002).

16. V. E. Panchuk, M. V. Yushkin, and I. D. Naŭdenov, SAO Preprint No. 179 (Special Astrophysical Observatory, 2003).

17. V. G. Klochkova, V. E. Panchuk, and M. V. Yushkin, SAO Preprint No. 168 (Special Astrophysical Observatory, 2002).

18. J. J. Michalsky, R. A. Stokes, and P. A. Ekstrom, Astrophys. J. 203, L43 (1976).

19. J. J. Michalsky, R. A. Stokes, and P. A. Ekstrom, Astrophys. J. 206, L73 (1976).

20. S. J. Shawl and M. Tarenghi, Astrophys. J. 204, L25 (1976)

21. T. L. Jones and H. M. Dyck, Astrophys. J. 220, 159 (1978).

22. P. G. Martin, Mon. Not. R. Astron. Soc. 159, 179 (1972).

23. P. G. Martin, R. Illing, and J. R. P. Angel, Mon. Not. R. Astron. Soc. 159, 191 (1972).

24. T. Gold, Mon. Not. R. Astron. Soc. 112, 215 (1952).

25. L. W. Bandermann and J. C. Kemp, Mon. Not. R. Astron. Soc. 162, 367 (1973).

26. R. D. Wolstenkroft and J. C. Kemp, Astrophys. J. 177, L137 (1972).

27. G. D. Schmidt, J. R. P. Angel, and E. A. Beaver, Astrophys. J. 219, 477 (1978).

28. D. A. Weintraub, J. H. Kastner, D. C. Hines, and R. Sahai, Astrophys. J. 531, 401 (2000).

29. M. Morris, Astrophys. J. 249, 572 (1981).

30. F. Yusef-Zadeh, M. Morris, and R. L. White, Astrophys. J. 278, 186 (1984).
31. R. Sahai, J. T. Trauger, A. M. Watson, et al., Astrophys. J. 493, 301 (1998).

32. R. Kawabe, M. Ishiguro, T. Kasuga, et al., Astrophys. J. 314, 322 (1987).

33. W. B. Latter, J. L. Hora, D. M. Kelly, et al., Astron. J. 106, 260 (1993).

34. M. Jura, S. P. Balm, and C. Kahane, Astrophys. J. 453, 721 (1995).

35. M. Jura, J. L. Turner, S. Van Dyk, and G. R. Knapp, Astrophys. J. 528, L105 (2000).

36. B. Lopez and J.-M. Perrin, Astron. Astrophys. 354, 657 (2000).

37. D. Crampton, A. P. Cowley, and R. M. Humphreys, Astrophys. J. 198, L135 (1975).

38. M. Cohen and L. V. Kuhi, Astrophys. J. 213, 79 (1977).

39. V. G. Klochkova, V. E. Panchuk, R. Szczerba, and K. Volk, Astron. Astrophys. 345, 905 (1999).

40. V. E. Panchuk, Candidate's Dissertation in Physics and Mathematics (Spets. Astrofiz. Obs. Ross. Akad. Nauk, Nizhniǔ Arkhyz, 1978), p. 167.

41. J. C. McCallum, W. R. Jarmain, and R. W. Nicholls, CRESS Spectroscopic Report: Franck-Condon Factors and Related Quantities for Diatomic Molecular Band Systems, No. 1, March 1970, York Univ.

42. M. Cohen and L. V. Kuhi, Publs Astron. Soc. Pac. 92, 736 (1980).

43. D. L. Lambert, Y. Sheffer, A. C. Danks, et al., Astrophys. J. 353, 640 (1990).

44. R. Gredel, E. F. van Dishoeck, and J. H. Black, Astrophys. J. 338, 1047 (1989).

45. B. J. Hrivnak, S. Kwok, and K. Y. L. Su, Astron. J. 121, 2775 (2001).

46. P. Cox, R. Lucas, P. J. Huggins, et al., Astron. Astrophys. 353, L25 (2000).

47. C. J. Skinner, M. Meixner, M. J. Barlow, et al., Astron. Astrophys. 328, 290 (1997).

Translated by N. Samus' 